\title{
Abdominal Pregnancy, Case Report from Latifa Hospital DHA, Dubai, UAE
}

\author{
Atif Bashir E. Fazari1,2*, Vidya Raman', Hala Bashir'1, Faiqha M. Azim¹, Sawsan Kh. Halou1 \\ ${ }^{1}$ Latifa Hospital, Dubai Health Authority, Dubai, UAE \\ ${ }^{2}$ Faculty of Medicine, University of Medical Sciences \& Technology, Khartoum, Sudan \\ Email: *atiffazari@hotmail.co.uk
}

How to cite this paper: Fazari, A.B.E. Raman, V., Bashir, H., Azim, F.M. and Halou, S.Kh. (2018) Abdominal Pregnancy, Case Report from Latifa Hospital DHA, Dubai, UAE. Open Journal of Obstetrics and Gynecology, 8, 1198-1204.

https://doi.org/10.4236/ojog.2018.812121

Received: September 25, 2018

Accepted: October 21, 2018

Published: October 24, 2018

Copyright $\odot 2018$ by authors and Scientific Research Publishing Inc. This work is licensed under the Creative Commons Attribution International License (CC BY 4.0).

http://creativecommons.org/licenses/by/4.0/

\begin{abstract}
Abdominal pregnancy is a rare form of ectopic pregnancy. A high index of suspicion enables early diagnosis and prompt management. Ultrasound is a gold standard tool for diagnosis and Magnetic Resonance imaging has a limited role. Appropriate management of the condition is important to avoid grave maternal morbidity.
\end{abstract}

\section{Keywords}

Abdominal Pregnancy, Ectopic Pregnancy, Fetal Morbidity, Maternal Mortality

\section{Introduction}

Abdominal pregnancy is a potentially life threatening form of ectopic gestation, with an incidence of $1 \%-1.4 \%$ [1]. Advances in prenatal care and imaging studies in the last few decades have helped early diagnosis and management. The clinical features vary based on site and gestation at presentation. A high index of suspicion enables early diagnosis and prompt management. Ultrasound remains the primary means of diagnosis. Surgical intervention is the ideal best option for treatment. Timed diagnosis and prompt management minimize maternal morbidity and prevent mortality. We reported this case to study diagnosis, management and outcome of abdominal pregnancy.

\section{Case Report}

A 26-year-old lady with no past significant medical or surgery history, para 1, previous normal vaginal delivery 10 years back, presented to the emergency department at Latifa Hospital, Dubai Health Authority, Dubai, UAE with com- 
plaints of spotting and bleeding per vagina for the past 2 days, which was unprovoked and painless. Her menstrual cycles were irregular and she was not sure of her last menstrual period, but she gave a history of amenorrhea of $6-7$ months. A urine pregnancy test done by her at home was positive three months back. She had not seeked any medical care for confirming her pregnancy or any obstetric care. She claimed to have fetal movements until the previous day of her visit to the hospital. She had no other associated symptoms and no other significant history.

Upon arrival in hospital, she was conscious and oriented, comfortable and vitally stable with a heart rate of $79 \mathrm{bpm}$, blood pressure of $123 / 83 \mathrm{mmHg}$, oxygen saturation of $100 \%$, respiratory rate was 13 and with temperature of $36.9^{\circ} \mathrm{C}$. Her abdomen was soft and non-tender, with a fundal height corresponding to 26 weeks of gestational age. Fetal heart could not be heard with a hand held doptone.

A bed side scan at the emergency department revealed only a slightly enlarged uterus with empty uterine cavity and thick endometrium. A fetus was seen above the uterus with a femur length of 22+ weeks and no fetal heart. A mass also seen in the right adnexa, which was difficult to describe.

An empirical diagnosis of an abdominal pregnancy with a non-viable fetus of $22+$ weeks was made and this was confirmed by an urgent abdominopelvic scan done in radiology department. The uterus was empty with a homogeneous echotexture and blood clots of $4.9 \times \mathrm{cm}$, Figure 1. Extra uterine pregnancy cephalic to the uterus with a collapsed fetus near the liver, with biometric parameters of $22+6$ weeks without fetal heart activity was seen, Figure 2 and Figure 3 . Caudal to the collapsed fetus, a placenta was seen measuring $13.4 \times 9.5 \times 7 \mathrm{~cm}$, Figure 4. Left ovary was normal and right ovary was not seen. Minimal free fluid in the pouch of Douglas.

Blood biochemistry and hematology reports were all within normal ranges with a hemoglobin of $11.7 \mathrm{gm} / \mathrm{dl}$, total white blood cells of $8.710^{3} / \mathrm{uL}$ and normal platelet count. Blood group and $\mathrm{Rh}$ type was $\mathrm{AB}$ positive. Blood urea, serum electrolytes and coagulation profiles were within normal limits.

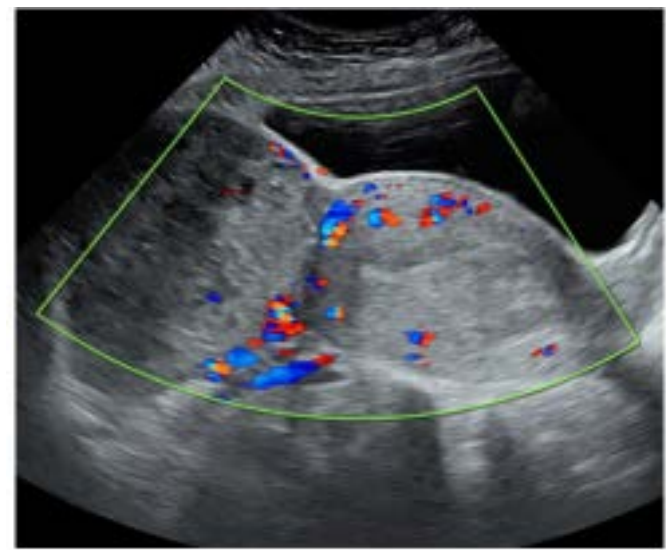

Figure 1. Empty uterine cavity. 


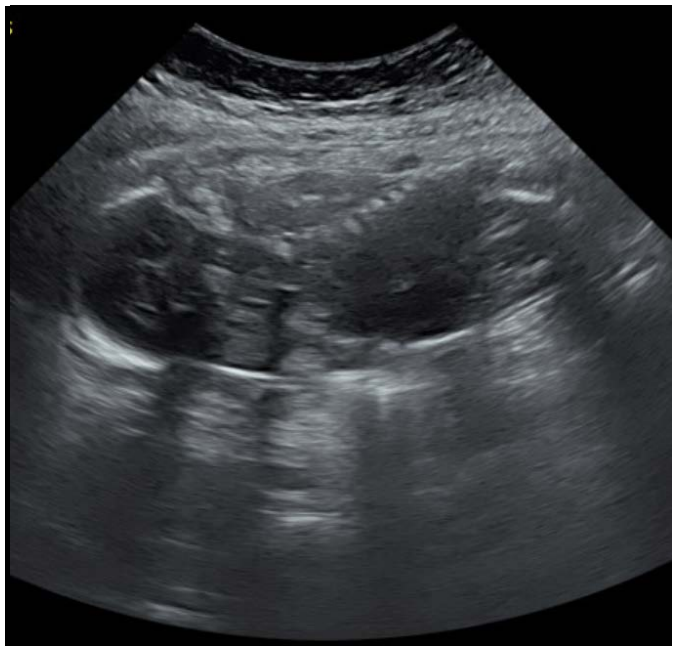

Figure 2. The collapsed fetus.

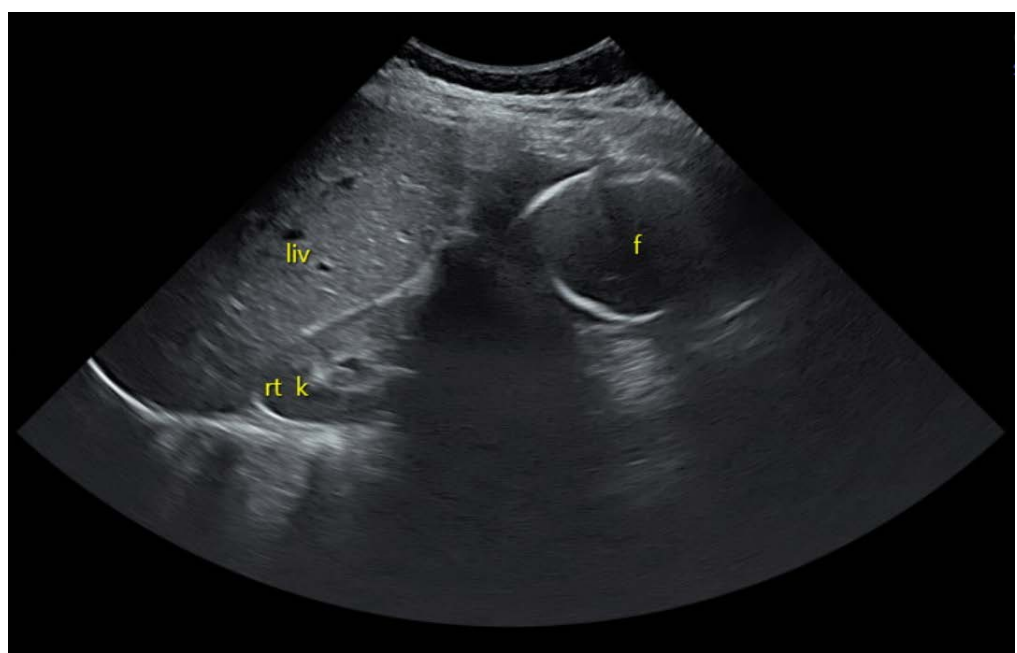

Figure 3. The collapsed fetus (f) near the liver (liv) and the right kidney (rt, k).

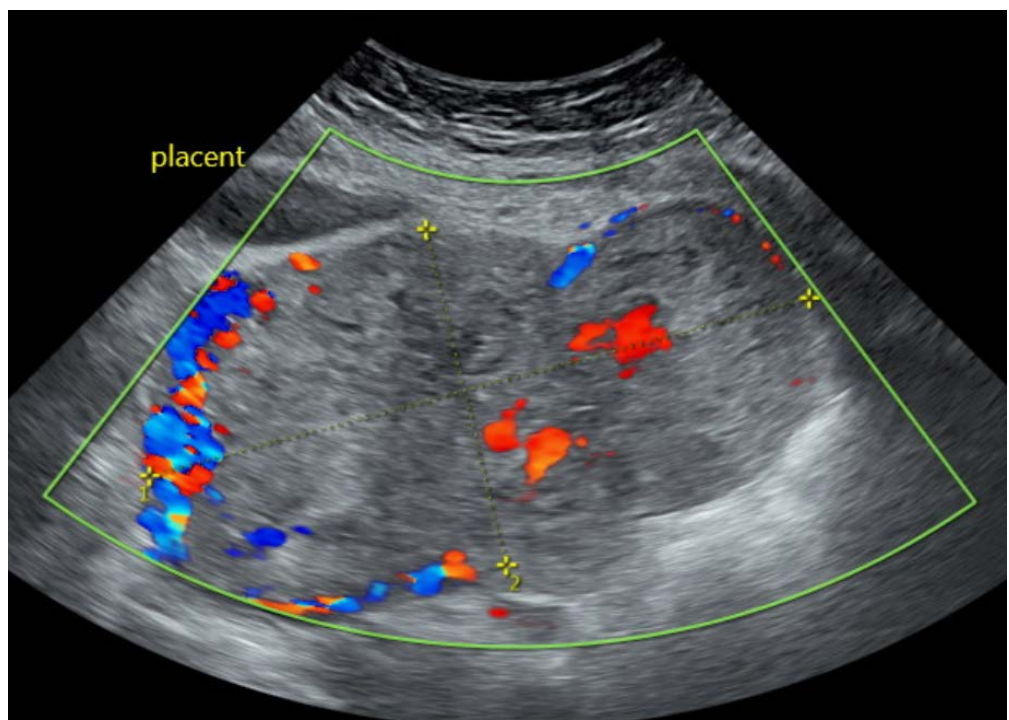

Figure 4. The placenta with Doppler study. 
After admission, she was monitored for any deterioration of clinical condition. Patient was counselled about the diagnosis and plan of management. All the possible complications: bleeding, infection, Disseminated Intravascular Coagulopathy, involvement of abdominal viscera, bowel resection, end-to-end anastomosis, colostomy, bladder involvement, and damage well explained. She was very understanding and agreed to the plan of management. A written consent was taken and the patient prepared for surgery. A laparotomy was planned. The general surgeon and urologist kept informed. Adequate blood and blood products were kept ready.

A laparotomy with midline vertical incision was performed. A lobulated mass measuring around $22 \mathrm{~cm} \times 12 \mathrm{~cm}$ was seen sitting on the right cornea and lateral pelvic wall. The umbilical cord could be seen running from this mass upwards into the bowel loops and omentum. The mass was removed enblock along with the right adnexa, lateral to the infundibulo-pelvic ligament. The fetus was then traced along the cord which was under the right liver lobe with its head towards the porta hepatis. With careful dissection, the fetus was removed ensuring safety and adequate hemostasis. The omentum had large hemorrhagic areas, which necessitated omentectomy, Figure 5. Adequate hemostasis secured with additional sutures and cautery. The bowel, others intraabdominal organs, ureters were all thoroughly examined; no damage or bleeding anywhere was detected, thorough warm saline wash given. An intraperitoneal drain was left and abdomen closed in layers. Intraoperative blood loss was estimated to be around $400 \mathrm{ml}$.

Post operatively she had a smooth, uneventful recovery. An ultrasound scan was repeated on the third day which showed a normal pelvi-abdominal cavity and no intraperitoneal collection. She was discharged home on the fourth day with a follow up appointment in 4 weeks showed healthy scar and no complaint. On the 3 months follow up visit she quite well, healthy and no any complaint.

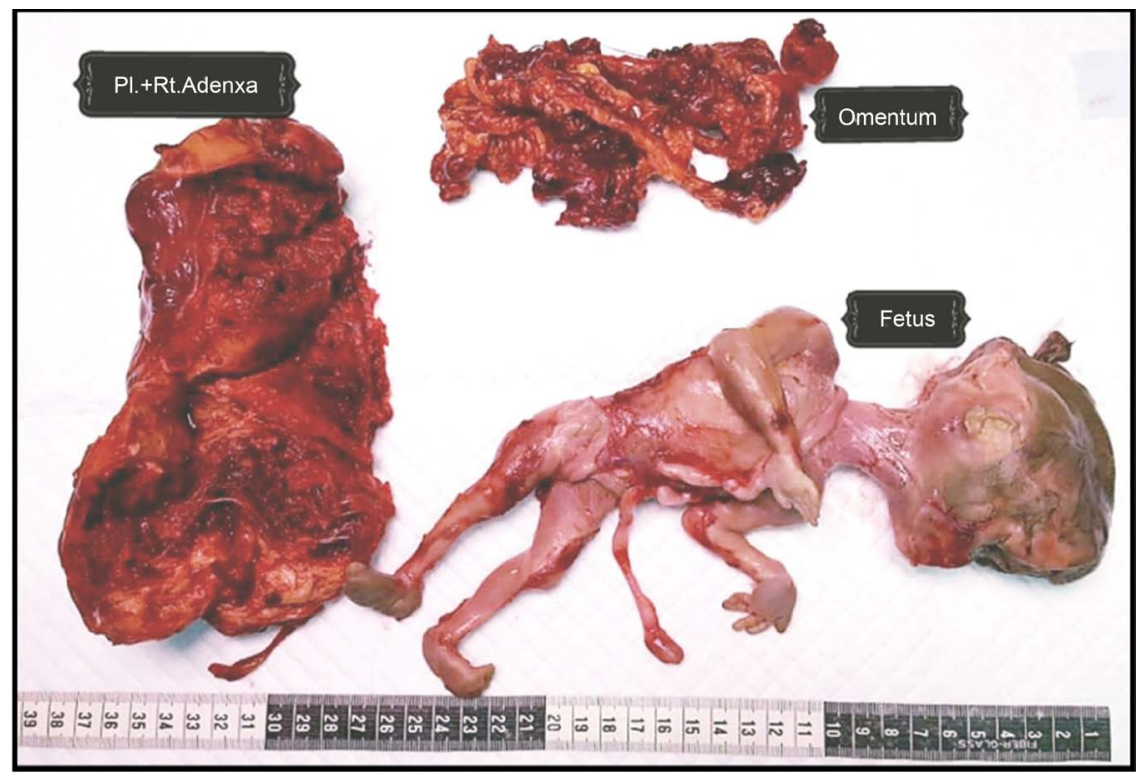

Figure 5. The fetus, placenta and piece of Omentum. 


\section{Discussion}

Abdominal pregnancy is an ectopic pregnancy where the fetus and placenta is implanted in the peritoneal cavity. Implantation in the ovary, tubes and the broad ligament is not considered as abdominal pregnancy by several experts.

It is said to be primary, when the implantation occurs directly in the peritoneum. Such pregnancies are very rare. More commonly, implantation is secondary in which initially the pregnancy gets implanted in the tube or ovary which later gets re-implanted in the peritoneum. This could happen because of a fimbria abortion, or rarely due to uterine rupture from previous scar or from a rudimentary horn of the uterus. A rare case following perforation of uterus during embryo transfer during IVF has also been reported [2]. Implantation could be in any abdominal peritoneum like the pelvic side walls, parietal peritoneum over the uterus, the pouch of Douglas, omentum, bowel and mesentery, mesosalpinx etc. Rare instances of implantation over the liver, spleen and under surface of the diaphragm have also been described [3].

Multiparity, tubal damage due to pelvic inflammatory disease and endometriosis and previous tubal surgery are risk factors just like for any other ectopic pregnancy. Smoking is also a significant risk factor. In more than half of the cases, no obvious risk factor might be detected.

Women usually present with abdominal pain and vaginal bleeding during pregnancy like our case, gastrointestinal symptoms, and painful fetal movements which is not seen here lucky enough because of fetal demise. Findings like abnormal lie and presentation, easily palpable fetal parts, uneffaced cervix at term should raise a suspicion. Term pregnancies with failed inductions have been taken for cesarean sections and the surgeons found intraabdominal pregnancy as an unexpected diagnosis. Acute presentation due to intraabdominal bleeding leading to severe abdominal pains, hypovolemia and shock and its complications have also been reported.

A high index of suspicion enables early diagnosis and prompt management. Ultrasound remains the primary means of diagnosis; it can be frequently missed, depending upon the operator's experience. Presence of features like an empty uterus reduced amniotic fluid, absent uterine wall around the fetus, with abnormal lie, abnormal looking placenta with free fluid in the peritoneal cavity help in clinching the diagnosis. Apart from this, ultrasound would also help detect fetal anomalies which are high in abdominal pregnancies.

Ultrasound diagnosis of the rare primary abdominal pregnancy needs the fulfillment of the Studiford's criteria, which are as follows:

- Normal looking tubes and varies.

- No connection or fistula between the uterus and abdominal cavity.

- Thirdly, pregnancy should be related only to the peritoneal cavity without signs that it was a tubal pregnancy at any time [4].

Magnetic Resonance Imaging (MRI) has a limited role to play. It aids in better imaging of the fetus and placental location, and attachment to visceral organs to plan surgery for the woman. MRI or Doppler Ultrasound could be used to local- 
ize the placenta [5]. Here Doppler Ultrasound was used to detect the placenta perfectly it localized the placenta, Figure 4 Elevated levels of maternal serum and amniotic fluid alpha-feto proteins also is associated with the condition.

\section{Treatment}

Appropriate management of the condition is important to avoid grave maternal morbidity. The patient has to be adequately counseled about the condition, outcome, associated and expected complications with regards to placental attachment, bleeding and involvement of viscera. Procedures like viscrectomy, bowel resection with anastomosis or colostomy, bladder injury is one of reported complication. In early abdominal pregnancies identified before 20 weeks of gestation, surgical termination is the mainstay of treatment. Laparotomy or laparoscopic methods can be chosen depending upon the expertise available [5].

Use of methotrexate is also an option, but the bulk of tissue should always be kept in mind, for necrosis of a larger tissue mass can lead to infection and sepsis and hence is not advisable.

Surgery is treatment of choice in advanced abdominal pregnancy beyond 20 weeks of pregnancy. Management of the placenta poses a challenge to the surgical team. Measures should be in place to manage torrential life threatening bleeding. The umbilical cord can be ligated and left the placenta in situ and monitored for spontaneous involution.

Alternatively, methotrexate or vessel embolization can be used to hasten the process of involution. Serial ultrasound scans and beta hCG levels can guide in monitoring the process of placental resorption which can sometimes take up to years. She should also be monitored for inflammatory changes related to necrosis, like bowel and ureteric obstruction and fistula formation, bleeding, infection and sepsis.

There is a role for conservative management in select cases, diagnosed above 24 weeks, in order to achieve viability This is possible only in centers where there are facilities for close inpatient monitoring, ready availability of surgical intervention and blood transfusion services.

There have been reports of abdominal pregnancies continuing up to term. Rarely, a calcified fetus can be found in the peritoneal cavity years after the index case, which is called a lithopedion [4] [5].

\section{Conclusion}

Abdominal pregnancy is a rare form of ectopic pregnancy. It carries high maternal morbidity and fetal morbidity. Abdominal pregnancy is challenging for the attending medical team in terms of diagnosis, timely intervention and surgical procedure.

\section{Acknowledgements}

All thanks to Latifa Hospital administration, Obstetrics \& Gynecology depart- 
ment, Anesthesia department, Theater staff and the Nursing staff for their unlimited cooperation and outstanding care in cases management.

\section{Ethical Approval}

Ethical clearance is obtained from the patient herself by written and informed consent as well from Obstetrics \& Gynecology department.

\section{Conflicts of Interest}

No conflict of interest.

\section{References}

[1] Gibbs, R.S. (2008) Danforth's Obstetrics and Gynecology. 10th Edition, Lippincott Williams \& Wilkins, Philadelphia, 84.

[2] Yoder, N., Tal, R. and Martin, J.R. (2016) Abdominal Ectopic Pregnancy after In Vitro Fertilization and Single Embryo Transfer: A Case Report and Systematic Review. Reproductive Biology and Endocrinology, $R B \& E, 14,69$. https://doi.org/10.1186/s12958-016-0201-x

[3] Chui, A.K., Lo, K.W., Choi, P.C., Sung, M.C. and Lau, J.W. (2001) Primary Hepatic Pregnancy. ANZ Journal of Surgery, 71, 260-261. https://doi.org/10.1046/j.1440-1622.2001.02085.x

[4] Berek, J.S. (2007) Berek \& Novak's Gynecology. 14th Edition, Lippincott Williams \& Wilkins, Philadelphia, 628.

[5] Bertrand, G., Le Ray, C., Simand-Emond, L., Dubois, J. and Leduc, L. (2009) Imaging in the Management of Abdominal Pregnancy: A Case Report and Review of the Literature. Journal of Obstetrics and Gynaecology Canada, 31, 57-62. https://doi.org/10.1016/S1701-2163(16)34055-5 